\section{Aducanumab: falta de consistencia entre la evidencia preclínica y clínica}

\section{Aducanumab: Lack of consistency between preclinical and clinical evidence}

\section{Señor Editor,}

Actualmente, Chile es un país envejecido, y entre los retos a los sistemas de salud pública relacionados con la edad destaca el aumento de la prevalencia de demencias entre las personas mayores. Las demencias son condiciones de alta complejidad en su enfrentamiento, con severas consecuencias tanto para los pacientes como para las familias afectadas. Entre sus causas sobresale la enfermedad de Alzheimer (EA) y los trastornos relacionados. En ese sentido, las terapias que logren modificar el curso de esta enfermedad, ausentes en el arsenal terapéutico actual, son un gran desafío de investigación y constituyen uno de los hitos más esperados de la ciencia a nivel mundial.

Recientemente, la oficina estadounidense de Administración de Medicamentos y Alimentos (FDA, por sus siglas en inglés) informó la aprobación del fármaco aducanumab (Aduhelm), sintetizado por la empresa de biotecnología Biogen. La molécula activa es un anticuerpo dirigido contra el péptido beta amiloide y su aprobación ha generado controversia ya que, a juicio de la comunidad científica, carece de suficiente evidencia clínica que asegure su eficacia y, adicionalmente, se han observado algunos efectos adversos ${ }^{1}$.

El sitio de acción del aducanumab es el péptido beta amiloide, un fragmento de la proteína precursora amiloide que se acumula en el cerebro de pacientes con EA, desencadenando una serie de procesos y eventos tóxicos que llevan a la muerte neuronal (neurodegeneración) y clínicamente a la demencia. Esto se conoce como la "hipótesis amiloide", una teoría con gran sustento científico que explica que un aumento en la producción del péptido lleva a su acumulación en las placas amiloides, agregado insoluble que se deposita en áreas relevantes para la función cognitiva. Este fragmento proteico sería el iniciador de la formación de ovillos neurofibrilares y la pérdida de conectividad neuronal, inflamación, daño oxidativo y neurodegeneración que ocurre en las estructuras cerebrales.

Desde finales del siglo XX, en línea con esta hipótesis, diversos hallazgos en modelos animales de EA indicaron que la inmunización, tanto directa (el péptido o fragmentos de este) como pasiva (anticuerpos contra el péptido), lograba reducir los niveles del péptido amiloide y las placas amiloides en el cerebro, junto con una mejoría en el rendimiento conductual asociado al aprendizaje y la memoria. Esta evidencia promovió la realización de estudios clínicos, aplicando estrategias para estimular la respuesta inmune frente al péptido beta amiloide. Particularmente, el aducanumab es un anticuerpo monoclonal recombinante humano, es decir, sintetizado artificialmente ${ }^{1}$, específico para una región del péptido beta amiloide, que se une con alta afinidad a este, ya sea en agregados libres o solubles o formando parte de las placas amiloides. Estudios en modelos animales con administración intraperitoneal de aducanumab demostraron una reducción en el tamaño de las placas amiloides, pero no se observó efecto sobre el rendimiento cognitivo ${ }^{1}$.

Un primer estudio clínico de fase $\mathrm{I}^{2}$, utilizando dosis crecientes de aducanumab (10-60 mg/kg) administrado por vía endovenosa en individuos con EA leve a moderada, no mostró efectos en la evaluación cognitiva medidos con la escala Alzheimer's Disease Assessment Scale-Cognitive Subscale (ADAS-COG). En un segundo estudio de fase I (PRIME) ${ }^{1}$, desarrollado para evaluar la seguridad, tolerabilidad, farmacocinética y farmacodinamia de aducanumab en individuos con EA moderada se verificó, mediante tomografía por emisión de positrones, una disminución dosis-dependiente en la deposición amiloide y una atenuación en el declive cognitivo solo en algunas escalas de evaluación ( $M i$ ni-Mental State Examination, MMSE; Clinical Dementia Rating Scale-Sum of Boxes, CDR-SB). Sin embargo, en algunos casos, especialmente en portadores del alelo $\varepsilon 4$ de apolipoproteína $\mathrm{E}$, se presentó edema vasogénico y anormalidades neuroimagenológicas relacionadas con alteraciones amiloides (ARIA, por su sigla en inglés).

Posteriormente, Biogen llevó a cabo dos ensayos clínicos de fase III de mayor duración (ENGAGE y EMERGE) ${ }^{3}$, con mayor tamaño muestral que los previos, con individuos con EA moderado, examen neuroimagenológico (tomografía de emisión de positrones) y además portadores del alelo $\varepsilon 4$. Estos se iniciaron el 2015, y su término estaba programado para 2022. Sin embargo, los ensayos fueron suspendidos a comienzos de 2019, por la baja potencia estadística en la evaluación cognitiva, pese al reclutamiento de un grupo adicional de pacientes durante el estudio. No obstante lo anterior, en octubre de 2020 y de manera sorpresiva, Biogen anunció la solicitud de aprobación para la comercialización de aducanumab a la FDA, argumentando que al analizar los datos incluyendo a los participantes que continuaron con los ensayos se corroboraron hallazgos significativos, y un subgrupo de pacientes mostró un beneficio cognitivo. En estos estudios, la incidencia de ARIA fue dosis-dependiente y el efecto se observó en el grupo que recibió la dosis más alta ${ }^{4}$.

El 7 de junio de 2021, la FDA otorgó la aprobación al aducanumab como medicamento para el tratamiento de la $\mathrm{EA}^{5}$, a pesar de la recomendación de no aprobarlo realizada previamente por un comité asesor médico convocado por la misma agencia, debido a la inconsistencia de los resultados obtenidos en los estudios de 
fase III $^{6}$, pese a la plausibilidad biológica que podría suponerse al contemplar su mecanismo de acción. Ante la ausencia de evidencia científica suficiente sobre la eficacia del fármaco, este anuncio genera múltiples dudas en la comunidad médica y científica, al no posicionarse como una intervención con eficacia clínica demostrada. Adicionalmente, el tratamiento tiene un costo muy elevado, alcanzando USD $56000(€ 40000)^{7}$.

En suma, aunque los estudios disponibles indican que aducanumab tiene un efecto comprobado en la reducción de la carga amiloide cerebral, evaluada mediante neuroimágenes, creemos que esta aprobación debe tomarse con cautela ya que, a nuestro juicio, existe falta de consistencia entre la evidencia preclínica y clínica, y esta última es imprescindible para la aprobación del uso de una intervención farmacológica en seres humanos.

\section{Álvaro O. Ardiles ${ }^{1,2,3}$, Marcelo Arancibia ${ }^{2}$,} Mariane Lutz ${ }^{2,3}$, Julio Riquelme ${ }^{1}$, Juan Pablo Gigoux ${ }^{l}$, Pablo Muñoz ${ }^{1}$

${ }^{1}$ Centro de Neurología Traslacional,

Facultad de Medicina, Universidad de Valparaíso.

Valparaíso, Chile.

${ }^{2}$ Centro Interdisciplinario de Estudios en Salud

(CIESAL), Escuela de Medicina, Facultad de Medicina,

Universidad de Valparaíso. Viña del Mar, Chile.

${ }^{3}$ Centro Interuniversitario de Envejecimiento Saludable, Consorcio de Universidades del Estado de Chile (CUECH).

\section{Referencias}

1. Sevigny J, Chiao P, Bussière T, Weinreb PH, Williams L, Maier $\mathrm{M}$, et al. The antibody aducanumab reduces $\mathrm{A} \beta$ plaques in Alzheimer's disease. Nature [Internet]. 2016; 537
(7618): 50-6. Available from: https://pubmed.ncbi.nlm.nih. gov/27582220/

2. Ferrero J, Williams L, Stella H, Leitermann K, Mikulskis A, O’Gorman J, et al. First-in-human, double-blind, placebo-controlled, single-dose escalation study of aducanumab (BIIB037) in mild-to-moderate Alzheimer's disease. Alzheimer's Dement Transl Res Clin Interv [Internet]. 2016; 2 (3): 169-76. Available from: https://pubmed.ncbi.nlm.nih. gov/29067304/

3. Knopman DS, Jones DT, Greicius MD. Failure to demonstrate efficacy of aducanumab: An analysis of the EMERGE and ENGAGE trials as reported by Biogen, December 2019. Alzheimer's Dement [Internet]. 2021 [cited 2021 Jun 25];17(4):696-701. Available from: https://pubmed.ncbi. nlm.nih.gov/33135381/

4. Gleason A, Ayton S, Bush AI. Unblinded by the light: amyloid-related imaging abnormalities in Alzheimer's clinical trials. Eur J Neurol [Internet]. 2021; 28 (1): el. Available from: https://pubmed.ncbi.nlm.nih.gov/32808453/

5. Cavazzoni P. FDA's decision to approve new treatment for Alzheimer's disease [Internet]. 2021. Available from: https://www.fda.gov/drugs/news-events-human-drugs/ fdas-decision-approve-new-treatment-alzheimers-disease

6. Fillit H, Green A. Aducanumab and the FDA-where are we now? Nat Rev Neurol [Internet]. 2021; 17 (3): 129-30. Available from: https://pubmed.ncbi.nlm.nih.gov/33442064/

7. Fleck LM. Alzheimer's and aducanumab: Unjust profits and false hopes. Hastings Cent Rep [Internet]. 2021; Available from: http://doi.wiley.com/10.1002/hast.1264 